\section{Kariesexkavation + PolyBur}

\author{
Die Härte des PolyBur P1(Komet) ist geringer als gesundes und \\ höher als kariös verändertes Dentin. Damit soll der blaue Ro- \\ senbohrer bei der Exkavation helfen, das richtige Maß im pul- \\ panahen Bereich unter minimalinvasiven Ansprüchen besser \\ umzusetzen. In der Schwerpunktpraxis für Endodontologie \\ Dres. Käfferbitz, Hillscheid, ist es kein Widerspruch, durch den \\ zusätzlichen Einsatz des P1 alles zu tun, um einen endodonti- \\ schen Eingriff zu vermeiden.
}

\section{? Sie führen eine Schwerpunktpraxis für Endontologie ...}

Käfferbitz: ...ja, ich habe 2004/2005 als einer der Ersten das Curriculum Endodontologie über die apw absolviert. Seitdem widme ich mich in unserer 2-Behandlerpraxis zu über $30 \%$ endodontischen Behandlungsfällen, die ich aus der Region überwiesen bekomme.

? Wie sind Sie auf den PolyBur P1 aufmerksam geworden?

Käfferbitz: Bis vor kurzem stand ich wie viele Zahnärzte meiner Generation vor der Frage: Wie weit darf ich eigentlich exkavieren? Wo liegt der therapeutische Endpunkt? Als Student hatte ich gelehrt bekommen, dass der Kavitätenboden schön hart, weiß und beim Sondentest klirren soll. Doch die Lehre hat sich geändert. Im Sinne einer minimialinvasiven Zahnerhaltung versuchte ich zunächst den Kariesdetektor, der aber meines Erachtens immer noch zu viel Substanz forderte. Auf einer apw-Fortbidlungsveranstaltung 2012 machte mich schließlich ein Kollege auf den P1 von Komet aufmerksam.
? Wie gefiel Ihnen die Theorie, dass es einen selbstlimitierenden Bohrer gibt, der zwischen gesundem und krankem Dentin unterscheidet?

Käfferbitz: Die Idee fand ich klasse! Endlich war da ein Anhaltspunkt, ob ich noch weiter exkavieren muss oder ob ich schon aufhören darf. Typische Indikationen für den PolyBur P1 sind klinisch symptomlose Zähnen mit pulpanaher Kariesausdehnung, ausgedehnte Kavitäten beim „floriden“ Kariestyp und ausgedehnte Milchzahn-Kavitäten. Da der Hals des P1 sehr schlank ist, eignet er sich auch für Mikrokavitäten.

? Der Bohrer wird also immer zusätzlich eingesetzt und „vernudelt“, wenn er auf gesundes Dentin trifft. Wie viele PolyBurs brauchen Sie pro Zahn?

Käfferbitz: In der Regel nur 1! Sobald ich mich dem pulpanahen Gebiet nähere, wechsle ich vom Rosenbohrer aus Keramik (Komet CeraBur K1SM) oder Handexkavator zum P1. Ich setze ihn also sehr selektiv und punktuell im kritischen Gebiet ein. Vorzugsweise exkaviere ich dann mit

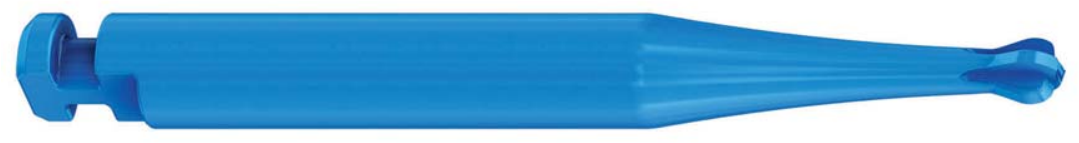

Abb 3 Die Indikation für den PolyBur P1 ist die weiche, pulpanahe Karies bei klinisch symptomlosen Milch- und bleibenden Zähnen.

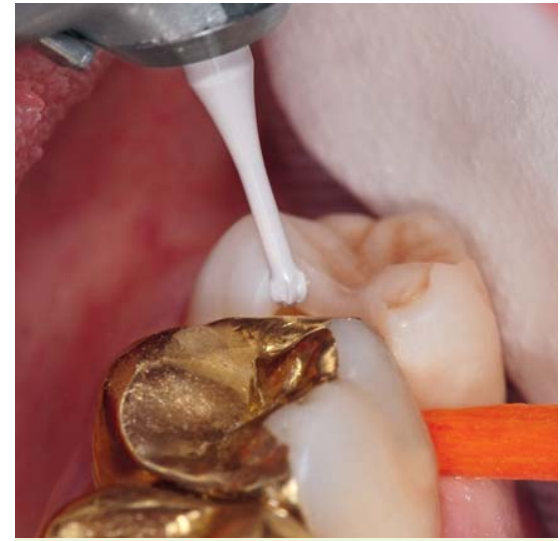

Abb1 Nach dem Eröffnen der Kavität mit Diamantinstrumenten werden die peripheren Anteile entfernt, z.B. mit dem CeraBur K1SM.

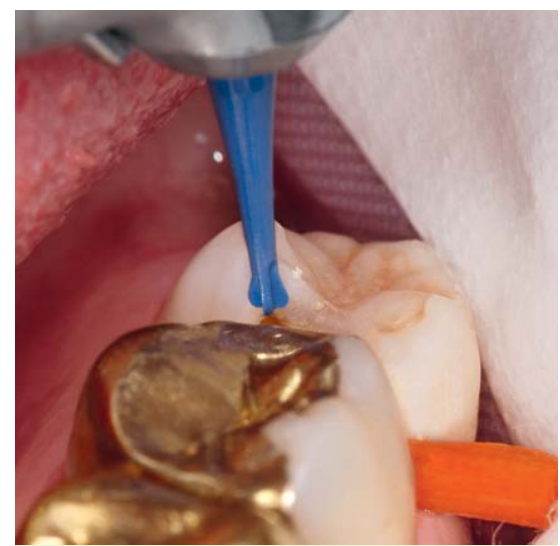

Abb 2 Pulpanahes Exkavieren mit dem PolyBur P1.

den großen Größen 018 und 023. In den seltenen Fällen, in denen eine Slotpräparation notwendig ist, greife ich auch ab und zu auf die Größe 014 zurück.

? Konnten Sie schon Endo-Patienten, die man Ihnen überwiesen hatte, Dank des Bohrers retten?

Käfferbitz: Ja, definitiv! Sie sind meist hoch erstaunt und glücklich, dass sie die Praxis nur mit einer Füllungstherapie wieder verlassen können, nachdem sie sich beim Besuch schon auf eine aufwändige Wurzelkanalbehandlung eingestellt hatten. Wenn ich aber mit dem P1 in die Pulpa einbreche, ist die endodontische Behandlung die logische Konsequenz. 
Käfferbitz: Ich gebe ganz offen zu: An die weichere Dentinoberfläche habe ich mich bis heute nicht gewöhnt. Laut Prof. Kunzelmann weist sie nur ca. 60 \% der Härte von kariesfreiem Dentin auf. Die Oberfläche erscheint nicht mehr so glatt und gut aussehend. Tja, da muss ich im Sinne einer modernen Zahnerhaltung durch. Sowohl die Überweiser als auch den Patienten muss ich jeweils über meine Vorgehensweise aufklären und sie darauf hinweisen, dass die Aufhellung im Röntgenbild keine Restkaries ist. Meist drücke ich dem Überweiser die Komet Produktinformation zum P1 in die Hand. Das hilft bei der Überzeugungsarbeit.

? Ohne Adhäsivtechnik würde Prof. Kunzelmann den P1 aber nicht anwenden!

Käfferbitz: Ein dichter Verschluss der Kavität ist eine wichtige Voraussetzung aller substanzschonenden Exkavationsverfahren. Deswegen versuche ich immer, die Kavitätenränder besonders sauber zu gestalten.

\section{Vielen Dank für das Gespräch.}

Das Interview führte Dorothee Holsten.

Kontakt

Zahnärzte Dres. Käfferbitz

Schwerpunkt Endodontie

Franz-Schubert-Str. 9

56204 Hillscheid

Dieser Beitrag entstand mit freundlicher Unterstützung von Komet Dental, Lemgo. 